\title{
ASPECTOS FUNDAMENTAIS PARA PROJETO E IMPLANTAÇÃO DE INSTALAÇÕES ELÉTRICAS HOSPITALARES
}

\author{
A. F. Spirandeli*1 ${ }^{*}$ S. F. P. Silva ${ }^{1}$, A. B. Soares ${ }^{1}$ \\ ${ }^{1}$ Faculdade de Engenharia Elétrica, Universidade Federal de Uberlândia, Uberlândia, MG, \\ Brasil. \\ *amanda.engbiomedica@gmail.com
}

\begin{abstract}
Resumo: Este trabalho é um documento objetivo sobre instalações elétricas com ênfase no ambiente hospitalar. Para realizar um projeto assim, é necessário entender a realidade e particularidades do ambiente, como os tipos de choque elétrico e os efeitos dos mesmos sobre o corpo humano, tipos de equipamentos, componentes do sistema elétrico, entre outras informações. Diante destas descrições, começa-se o levantamento do projeto em si, desde o fornecimento de energia dado pela concessionária local aos sistemas e componentes elétricos básicos, dando relevância à iluminação hospitalar, visto que é um aspecto que pode influenciar psicologicamente e fisiologicamente o quadro dos pacientes. Esse trabalho é de extrema responsabilidade para o auxílio de projeto, já que o ambiente hospitalar lida com situações críticas que requer segurança e confiabilidade.
\end{abstract}

Palavras-chave: Instalações elétricas, hospital, equipamentos eletromédicos.

\begin{abstract}
This work is an objective document on electrical installations with emphasis on hospital environment. To fulfill such a project, it is necessary to understand the reality and environmental characteristics, such as the types of electrical shock and their effects on the human body, types of equipment, electrical system components, among other information. Given these descriptions, the survey of the project itself is begun, since the power supply given by the local utility to systems and basic electrical components, giving relevance to the hospital lighting, once it is an aspect that can influence psychologically and physiologically the framework of patients. This work is of the utmost responsibility for project aid, as the hospital environment deals with critical situations that require security and reliability
\end{abstract}

Keywords: Electrical installations, hospital, medical electrical equipment.

\section{Introdução}

Projetar uma instalação elétrica, seja residencial, comercial ou industrial, consiste essencialmente em selecionar, localizar, e dimensionar, de maneira racional, os equipamentos e outros componentes necessários a fim de proporcionar, de modo seguro e efetivo, a transferência de energia da fonte até os pontos de utilização [1]. Tais questões tornam-se ainda mais relevantes ao se tratar do projeto de instalações em ambientes hospitalares, onde, além dos inúmeros equipamentos de alta sensibilidade, nos deparam com situações em que a monitoração e a manutenção da vida das pessoas depende do correto uso da eletricidade.

Diante disso, é importante mensurar e obter informações como o risco elétrico no uso da eletricidade no ambiente hospitalar englobando os tipos de choque e os efeitos fisiológicos que os mesmos podem causar ao ser humano. A fim de evitar circunstâncias de risco é necessários conhecer os tipos e medidas de proteção contra os choques elétricos, de acordo com as normas vigentes, para garantir confiabilidade e segurança ao ambiente, profissionais e pacientes.

O objetivo principal deste trabalho é definir e descrever os elementos essenciais a serem abordados por engenheiros biomédicos e eletricistas para o projeto e implantação de instalações elétricas particularidades ao ambiente hospitalar, de acordo com as normas vigentes.

\section{Materiais e métodos}

Para desenvolver um projeto de instalações elétricas de um ambiente hospitalar é necessário obter informações sobre os riscos da eletricidade, meios de proteção, equipamentos a serem utilizados, prática de instalação que abrangem em normas especificas e literaturas que serão abordadas a fim de pontuar a realização de um projeto. Assim, é importante avaliarmos os diversos efeitos da eletricidade no corpo humano e, posteriormente, os riscos associados ao ambiente hospitalar.

\section{Efeitos fisiológicos da eletricidade}

Para um efeito fisiológico ocorrer, o corpo deve tornar-se parte de um circuito elétrico. A corrente deve entrar em um ponto da matéria, neste caso o corpo, e deixá-la em algum outro ponto [2].

Webster [2], indica as intensidades de corrente necessárias para produzir distintos efeitos utilizando uma frequência de $60 \mathrm{~Hz}$, aplicada de 1 a 3 segundos, através de fios de cobre em cada mão, para um indivíduo com $70 \mathrm{~kg}$, conforme destacado na Fig. 1. 
- Threshold of perception (Limiar de percepção): nível de corrente mínima que pode ser detectado pela maioria das pessoas - 2 a $10 \mathrm{~mA}$ [2].

- Let-go current (Corrente let-go): valor máximo de corrente em que o sujeito pode retirar-se voluntariamente -6 a $90 \mathrm{~mA}[2]$.

- Respiratory paralysis, fatigue, pain (Paralisia respiratória, dor e fatiga): 18 a $22 \mathrm{~mA}$ [2].

- Ventricular fibrillation (Fibrilação ventricular): 75 à $400 \mathrm{~mA}$ [2].

- Sustained myocardial contraction (Contração miocárdica sustentada): 1 a 6 A (testes em animais) [2].

- Burns, injury (Queimadura e danos físicos): limiar de corrente $>10$ A [2].

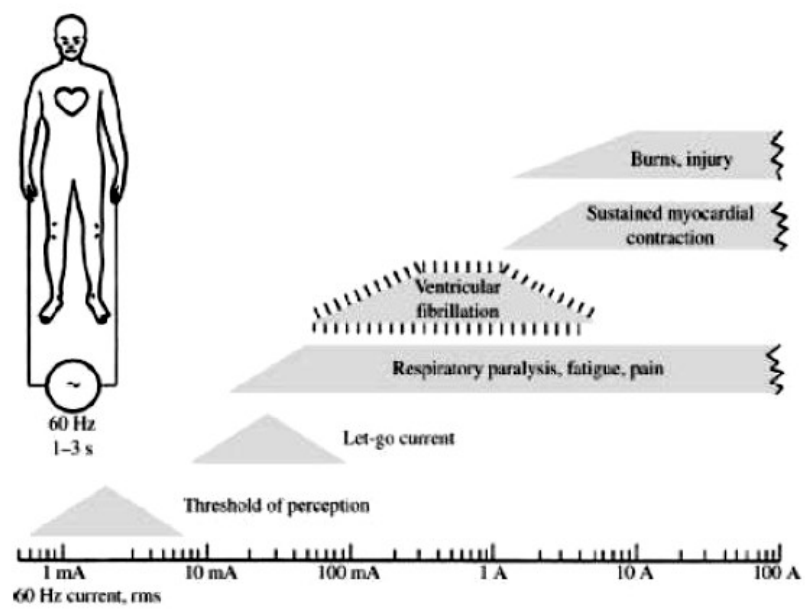

Figura 1: Efeitos Fisiológicos da Eletricidade [2].

\section{Origens do risco elétrico no ambiente hospitalar}

\section{Macro Choque}

O macro choque é o choque elétrico devido aos contatos estabelecidos externamente no corpo, com a pele intacta, e pode atingir tanto o paciente quanto o pessoal médico [3].

Alguns equipamentos eletromédicos possuem partes de metal, aos quais o pessoal médico e o paciente têm acesso. Caso estas partes de metal não estejam aterradas e ocorrendo um curto-circuito ou perda da isolação entre o condutor fase e as partes de metal, o indivíduo será submetido ao potencial da rede de alimentação. Caso o indivíduo toque, simultaneamente, na parte de metal do equipamento e em um objeto aterrado, ocorrerá um macro choque [3].

\section{Micro Choque}

O micro choque é o choque elétrico provocado no interior do organismo, proveniente de procedimentos invasivos, aplicado no coração ou próximo a ele, por meio de cateteres ou eletrodos [3].

Acidentes de micro choques em pacientes que possuem conexões elétricas diretas ao coração são normalmente causados por circunstâncias não relacionadas com riscos de macro choque [3].

\section{Proteção contra choques elétricos}

A proteção contra choques especificadas na norma [5] podem ser divididos em proteção básica, supletiva e adicional.

Proteção básica: meio destinado a impedir contato com partes vivas perigosas em condições normais, e corresponde ao conceito de proteção contra contato direto. Exemplos: isolação ou separação básica, uso de barreira ou invólucro e limitação da tensão [5].

Proteção supletiva: meio destinado a suprir a proteção contra choques elétricos quando massas ou partes condutivas acessíveis tornam-se acidentalmente vivas. Exemplos: equipotencialização, seccionamento automático da alimentação e isolação suplementar [5].

Proteção adicional: meio destinado a garantir a proteção contra choques elétricos em situações ou locais em que os perigos do choque elétrico são particularmente graves [5].

\section{Equipamentos eletromédicos}

Existem regras fundamentais aplicadas a equipamentos elétricos, e uma delas é que para a redução de riscos elétricos, todas as partes sob tensão estejam protegidas contra contatos acidentais. Tal regra permite distinguir os equipamentos eletromédicos em classes e tipos distintos, conforme o tipo e o grau de proteção contra choques elétricos.

\section{Classificação quanto ao tipo de proteção contra choques elétricos}

Equipamento classe I: além da isolação básica, incorpora um recurso de conexão do equipamento ao condutor de aterramento, para proteção pertencente à fiação fixa da instalação, de modo a impossibilitar que partes metálicas acessíveis possam ficar sob tensão, na ocorrência de uma falha na isolação básica [6].

Equipamento classe II: além isolação básica, incorpora ainda precauções de segurança adicionais, como isolação dupla ou isolação reforçada, não comportando recursos de aterramento para proteção, nem dependendo das condições de instalação [6].

\section{Classificação quanto ao grau de proteção contra choques elétricos}

Equipamento tipo F: a parte aplicada ou manipulada é separada eletricamente de todas as outras partes do equipamento [6].

Equipamento tipo B: proporciona um grau de proteção extra contra choque elétrico, particularmente quanto à corrente de fuga admissível e confiabilidade da conexão de aterramento para proteção (se existente) [6].

Equipamento tipo BF: equipamento tipo B com parte aplicada tipo $\mathrm{F}[6]$.

Equipamento tipo CF: proporciona um grau de proteção superior ao do equipamento de tipo BF contra 
choque elétrico, particularmente no que se refere às correntes de fuga admissíveis [6].

\section{Instalações elétricas hospitalares}

Segundo a norma [7], são três os grupos previstos para enquadramento dos recintos em um hospital em relação aos tipos de equipamentos eletromédicos a serem utilizados nos mesmo, ou que poderão vir a ser utilizados:

Recinto Grupo 0: recinto em que não são utilizadas partes aplicadas de equipamentos eletromédicos alimentados pela rede [7];

Recinto Grupo 1: recinto no qual se prevê o uso de equipamentos eletromédicos, mas não para procedimentos intracardíacos [7];

Recinto Grupo 2: recinto no qual se prevê o uso de equipamento eletromédico destinado a procedimento intracardíaco e equipamentos essenciais à manutenção da vida dos pacientes [7].

Ainda segundo a norma [7], outro elemento importante para a instalação elétrica hospitalar é a classificação da alimentação de segurança com que equipamentos do local em questão devem ser providos:

Classe 0,5: Alimentação automática disponível em até 0,5 segundo;

Classe 15: Alimentação automática disponível em até 15 segundos;

Classe >15: Alimentação disponível em mais que 15 segundos, em modo automático ou manual.

Observe que, em geral, não é necessário prover alimentação ininterrupta a equipamentos eletromédicos. Todavia, certos equipamentos podem exigir esta alimentação.

A Figura 2 representa o esquema básico de um sistema elétrico hospitalar em que se observam regiões com alimentação classe 15 e classe 0,5 . No diagrama podemos notar ainda os seguintes elementos clássicos:

IGURA 5.6 - Sistema elétrico hospitalar (G=gerador; $C . P=$ Concessionária púk

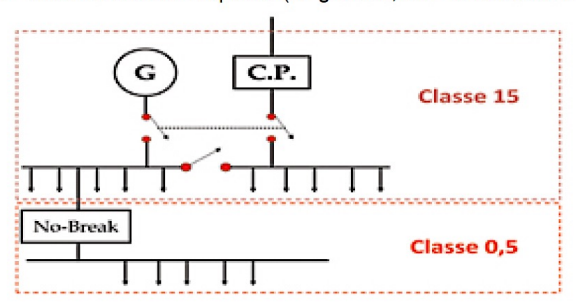

Fonte: Castellari (2016, p.34)

Figura 2: Sistema elétrico hospitalar $(\mathrm{G}=$ gerador; C.P $=$ Concessionária pública) [8].

- Fornecimento de energia (C.P.): concessionária local [8].

- Subestação Rebaixadora de Tensão: rebaixamento da tensão fornecida pela concessionária para as tensões de alimentação das cargas elétricas do hospital (380V, 220V e 127V) [8].

- Cogeração de Energia (G) - Sistema de energia elétrica de emergência: são compostos por grupos geradores diesel-elétrico (Classe 15) que possuem capacidade de fornecimento ininterrupto, porém, não apresentam condições de entrar no sistema imediatamente após a ocorrência da falta de energia, devido ao chaveamento, como pode ser observado na Figura 2, entram automaticamente em funcionamento em até 15 segundos [8].

- Cogeração de Energia (G) - Sistema de energia elétrica ininterrupta (Nobreak): são conectados à equipamentos que devem estar ligados no sistema de energia de emergência e, portanto, alimentados por duas fontes de energia, isto é, pela concessionária em regime normal e pelo sistema de geradores quando da falta de alimentação externa, conforme descrito na Figura 2. A energia fornecida pelo Nobreak deve ser de alta qualidade, isto é, sem variações de tensão, frequência e outros transtornos pertinentes do sistema elétrico convencional [8].

\section{Sistema It-médico}

Segundo a norma [7] o esquema IT-médico é um sistema de alimentação que não possui conexões direta entre partes sob tensão e aterramento, sendo as partes condutivas expostas na instalação elétrica aterrada. $\mathrm{O}$ esquema IT-médico utiliza transformador de separação elétrica para fins médicos aplicados em recintos de Grupo 2 e é composto por (Figura 3):

- Transformador de separação,

- Dispositivo supervisor de isolamento (DSI),

- Sistema de alarme.

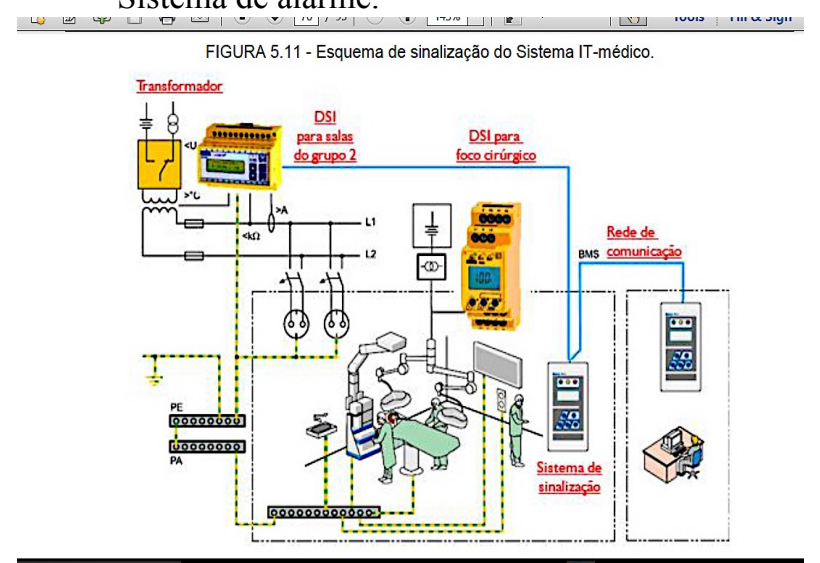

Figura 3: Esquema de sinalização do Sistema IT-médico [8].

\section{Componentes básicos do sistema elétrico no ambiente hospitalar}

Ao projetar as instalações elétricas para sistemas hospitalares, deve-se atentar diversos aspectos, em especial:

- Linhas elétricas e condutores 
Conforme a norma [5], em locais de afluência de público, as linhas elétricas embutidas devem ser totalmente imersas em material incombustível.

\section{- Quadros de distribuição}

Os quadros de distribuição devem ser do tipo fechado, de modo a garantir o mínimo grau de proteção e devem ser instalados de forma a não permitirem acesso involuntário do público. No caso do Sistema ITmédico deve-se ter um quadro separado.

\section{- Pontos de tomada}

A norma [9], especifica os seguintes números de tomadas para ambientes hospitalares:

- Unidade de internação geral e berçários: uma tomada para cada equipamento biomédico, por leito, e de uma tomada para acesso do aparelho de raios $X$ transportável distante no máximo $5 \mathrm{~m}$ de cada leito [9];

- Berçário de cuidados intermediários: pelo menos três tomadas para cada berço/Incubadora [9];

- Quartos e áreas coletivas da Unidade de Internação Intensiva: oito tomadas [9];

- Sala de cirurgia e sala de parto: são necessários dois conjuntos com quatro tomadas, cada um em paredes distintas, e também uma tomada por aparelho transportável de raios X [9].

\section{- Especificações para pontos de iluminação}

- Quartos enfermaria, quartos e área coletiva da UTI [9]:

- iluminação geral em posição que não incomode o paciente deitado;

- iluminação de cabeceira de leito na parede (arandela) para leitura;

- iluminação de exame no leito com lâmpada fluorescente;

- iluminação de vigília na parede (a $50 \mathrm{~cm}$ do piso).

- Sala de cirurgia e sala de parto: iluminação geral de teto com lâmpada fluorescente e a iluminação direta com foco cirúrgico [9].

- Consultórios e salas para exames clínicos: iluminação que não altere a cor do paciente [9].

\section{Resultados e discussão}

Diante de alguns requisitos essenciais para projetar as intalações elétricas de um ambiente hospitalar, e das prescrições das normas vigentes, este artigo aponta as os principais elementos e preceitos a serem observados por engenheiros biomédicos, eletricistas e arquitetos e demais profssionais envolvidos no projeto. Buscou-se apontar os principais fatores a serem considerados, desde $o$ fornecimento de energia entregue pela concessionária local, até os pontos de utilização especificos para cada setor, conforme suas classificações.

É importante observar que o projetista deve sempre buscar fazer um "check list", reunindo informações do cliente, bem como as descrições da demanda de cada setor e equipamentos à ser instalado, e associá-las aos projetos de arquitetura, construção civil, hidráulico, de gases, entre outros, indispensáveis para tal ambiente. Além disso, tais elementos de projeto devem ser apresentados à diversos órgão e pessoal envolvido, como conselheiros da instituição, diretores e projetistas, para só então, mediante sua aprovação, finalizar todos os elementos de projeto, em especial aqueles associados às instalações elétricas.

\section{Conclusão}

Conforme descrito ao longo deste artigo, as demandas específicas associadas ao projeto de instalações elétricas hospitalares podem ser muito complexas e demandam especial atenção dos projetistas, em especial engenheiros biomédicos e eletricistas. Tais profissionais devem buscar trabalhar com máxima harmonia e sinergia de modo a garantir o projeto adequado das diversas instalações voltadas ao atendimento médico de pacientes.

\section{Referências}

[1] Cotrim A. A. M. B. Instalações elétricas. 5th ed. São Paulo: Pearson Prentice Hall, 2009.

[2] Webster, J. G. Medical instrumentation: applications and design. 4th ed. New York: John Willey \&Sons, 1998.

[3] Dalziel, C. F. Effects of Electrical Shock on Man. Electrical Engineering. Geneva: International Labor Office, 1961.

[4] Associação brasileira de normas técnicas. NBR 5410: Instalações elétricas de baixa tensão. 2th ed. Rio de Janeiro: Abnt, 2004.

[5] Associação brasileira de normas técnicas. NBR IEC 601-1: Equipamento eletromédicos - Parte 1 Prescrições para segurança. [s.n.]. Rio de Janeiro: Abnt, 1996.

[6] Associação brasileira de normas técnicas. NBR 13534: Instalações elétricas para estabelecimentos assistenciais de saúde - Requisitos de segurança. [s.n.]. Rio de Janeiro: Abnt, 1995.

[7] Coutinho A. O papel da energia elétrica nos hospitais. [s.1., s.n., 20--].

[8]Brasil. Ministério da saúde. Agência Nacional de Vigilância Sanitária. Resolução RDC no 50. Brasília: Diário Oficial da União da República Federativa do Brasil 2002. 